\title{
Review of: "Estimated Fraction of Incidental COVID Hospitalizations in a Cohort of 250 High-Volume Hospitals Located in 164 Counties"
}

\author{
Mario Saia, Claudio Barbiellini Amidei
}

Potential competing interests: The author(s) declared that no potential competing interests exist.

At a population level, a better understanding of what fraction of hospitalizations are due to symptomatic COVID-19, as opposed to those hospitalized with a SARS-CoV-2 infection, are of great relevance for public health experts and remain a topic of open debate. As the pandemic is progressively slowing down, assessing the actual impact of COVID-19 on hospitals and healthcare systems is still warranted, as well as retrospective analyses focusing especially on end December 2021 and January 2022 when an enormous amount of patients were infected with SARS-CoV-2, and especially the Omicron Variant, without having any clinically relevant COVID-19 symptoms. Pre-established criteria to estimate the fraction of incidental COVID-19 cases are missing, and the presence of numerous variants characterized by different grades clinical severity render its correct quantification even more complex.

In a recent article, Dr. Jeffrey E. Harris proposed a formula to estimate the fraction of incidental COVID-19 hospitalizations starting from measurable population-based parameters. Analyzed data consists in a longitudinal panel of 164 counties throughout the United States, covering a 4-week interval ending the first week of January 2022 [Jeffrey E. Harris 2022]. During the study period, reported rates of infections rose exponentially at 9.34\% per day (95\% Cl, 8.93-9.87). By assuming a possible under-notification with only one-quarter of all Omicron infections being correctly identified, aggregated prevalence of active SARS-CoV-2 infection during the first week of January was estimated to be $4.89 \%$. The author reports that among 250 high-COVID-volume hospitals, about 1 in 4 patients tested positive, corresponding to an estimated incidental fraction of $15.2 \%$. When examining data across individual counties, the estimated median fraction of incidental COVID hospitalizations was $13.7 \%$, with an interquartile range of $9.5-18.4 \%$. The author then concluded that incidental SARS-CoV-2 infections appeared to be a nontrivial fraction of all hospitalized patients that tested positive. However, the burden of patients admitted for complications of their viral infections seemed to be significantly greater.

This study provides a useful tool to estimate the actual burden on hospitals attributable to COVID-19. Nevertheless, a validation process of this method is warranted, as well as a test to assess its accuracy in other countries. Given the possibility of future pandemic waves, this and similar approaches tailored on specific healthcare systems, and specific characteristics of SARS-CoV-2 variants could be of great use for policy makers. 